\title{
Combined effects of thermal treatment and Er-substitution on phase formation, microstructure, and dielectric responses of Bi4Ti3O12 Aurivillius ceramics
}

\begin{abstract}
The combined effects of thermal treatment and single erbium $(\mathrm{Er} 3+)$ ion-substitution $(\mathrm{x}=$ 0.75) on the A-site of Bi4-xErxTi3O12 compounds were investigated by scrutinizing the crystal structure, microstructural evolution, and dielectric properties. The results are compared with those of pure Bi4Ti3O12 compounds. Upon doping, the crystal structures experienced strong octahedral distortions, resulting in phase transformation from orthorhombic to monoclinic symmetry. The doped samples became highly anisotropic with increasing sintering temperature, which are accompanied by negative thermal expansion. Following the phase transformation, plate-like grains emerged at the expense of equiaxed grains, becoming more dominant as grain growth increased with sintering temperature. The grain growth rate is comparatively slower in the doped samples, implying the inhibiting role of Er. The doped samples showed relatively small loss of $\varepsilon$ " characterized by shorter relaxation times, which compromised the dielectric constant $\varepsilon^{\prime}$ due to the suppression of oxygen vacancies that is closely related to the smaller grain sizes and densities. Both $\varepsilon^{\prime}$ and $\varepsilon$ " of pure and doped samples are grain size dependent, in which the frequency responses followed the superposition of Cole-Cole and Havriliak-Negami distribution functions that correlates with the impedance studies.
\end{abstract}

Keyword: Ferroelectrics; High-energy ball milling; X-ray diffraction; Dielectric response 